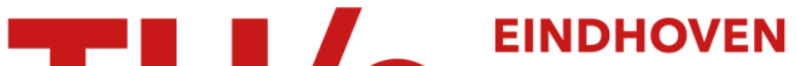 UNIVERSITY OF TECHNOLOGY
}

\section{Self-stresses control stiffness and stability in overconstrained disordered networks}

Citation for published version (APA):

Bose, A., Vermeulen, M. F. J., Storm, C., \& Ellenbroek, W. G. (2019). Self-stresses control stiffness and stability in overconstrained disordered networks. Physical Review E, 99(2), [023001].

https://doi.org/10.1103/PhysRevE.99.023001

DOI:

10.1103/PhysRevE.99.023001

Document status and date:

Published: 12/02/2019

\section{Document Version:}

Publisher's PDF, also known as Version of Record (includes final page, issue and volume numbers)

\section{Please check the document version of this publication:}

- A submitted manuscript is the version of the article upon submission and before peer-review. There can be important differences between the submitted version and the official published version of record. People interested in the research are advised to contact the author for the final version of the publication, or visit the $\mathrm{DOI}$ to the publisher's website.

- The final author version and the galley proof are versions of the publication after peer review.

- The final published version features the final layout of the paper including the volume, issue and page numbers.

Link to publication

\section{General rights}

Copyright and moral rights for the publications made accessible in the public portal are retained by the authors and/or other copyright owners and it is a condition of accessing publications that users recognise and abide by the legal requirements associated with these rights.

- Users may download and print one copy of any publication from the public portal for the purpose of private study or research.

- You may not further distribute the material or use it for any profit-making activity or commercial gain

- You may freely distribute the URL identifying the publication in the public portal.

If the publication is distributed under the terms of Article $25 \mathrm{fa}$ of the Dutch Copyright Act, indicated by the "Taverne" license above, please follow below link for the End User Agreement:

www.tue.nl/taverne

Take down policy

If you believe that this document breaches copyright please contact us at:

openaccess@tue.nl

providing details and we will investigate your claim. 


\title{
Self-stresses control stiffness and stability in overconstrained disordered networks
}

\author{
Anwesha Bose, ${ }^{1,}{ }^{*}$ Mathijs F. J. Vermeulen, ${ }^{1}$ Cornelis Storm,,${ }^{1,2}$ and Wouter G. Ellenbroek ${ }^{1,2, \dagger}$ \\ ${ }^{1}$ Department of Applied Physics, Eindhoven University of Technology, Den Dolech 2, 5600MB Eindhoven, The Netherlands \\ ${ }^{2}$ Institute for Complex Molecular Systems, Eindhoven University of Technology, Den Dolech 2, 5600MB Eindhoven, The Netherlands
}

(Received 2 December 2018; published 12 February 2019)

\begin{abstract}
We investigate the interplay between prestress and mechanical properties in random elastic networks. To do this in a controlled fashion, we introduce an algorithm for creating random free-standing frames that support exactly one state of self-stress. By multiplying all the bond tensions in this state of self-stress by the same number — which with the appropriate normalization corresponds to the physical prestress inside the frame-we systematically evaluate the linear mechanical response of the frame as a function of prestress. After proving that the mechanical moduli of affinely deforming frames are rigorously independent of prestress, we turn to nonaffinely deforming frames. In such frames, prestress has a profound effect on linear response: not only can it change the values of the linear modulus - an effect we demonstrate to be related to a suppressive effect of prestress on nonaffinity—but prestresses also generically trigger a bistable mechanical response. Thus, prestress can be leveraged to both augment the mechanical response of network architectures on the fly, and to actuate finite deformations. These control modalities may be of use in the design of both novel responsive materials and soft actuators.
\end{abstract}

DOI: 10.1103/PhysRevE.99.023001

\section{INTRODUCTION}

The concept of mechanical metamaterials [1-3]structures that inherit purposely targeted, nonstandard mechanical response to stress or strain from a particular spatial architecture, rather than from intrinsic properties of the materials they are composed of-has gained massive traction over the past decade. At macroscopic length scales, research into mechanical metamaterials has helped achieve, in systematic fashion, desirable properties such as auxeticity [4-8], chirality $[9,10]$, (origami-like) deployability and actuation [11-16], and anomalously high strength in ultra-light-weight lattices $[17,18]$. The central and profound insight that has enabled these breakthroughs is that the mechanical response of a generalized material is due to a combination of (i) the mechanical properties of its constituent(s), (ii) its spatial architecture (i.e., its void distribution), and (iii) its mechanical preconditioning, i.e., the configuration of internal stresses that reside on the spatial architecture. Each of these three factors may be targeted in design, but historically only (i) has been explored. Much of the current work in metamaterials can be understood as the exploration of a design space that stretches out along directions (ii) and (iii).

These concepts are simultaneously, and in parallel, finding their way into microscopic, molecular scale designs for polymeric matter where, likewise, they are allowing access to unusual mechanical response that is difficult to attain in pure bulk matter. Hydrogels, in particular, have proven to be a terrific canvas for exploring directions (ii) and (iii); harnessing

\footnotetext{
*a.bose@tue.nl

†w.g.ellenbroek@tue.nl
}

residual stresses, spatial composition, and noncovalent binding have yielded materials that, through purely mechanical and geometrical effects, exhibit greatly enhanced strength and toughness, structural adaptivity, and recyclability [19-25].

While the utility and successes of these microscopic design approaches in soft materials are irrefutable, a crucial difference between macroscopic and hydrogel metamaterials remains. Macroscopic materials are meticulously organized in space (by direct design of the entire structure or a unit cell), and they can be loaded at will. Smart hydrogel architectures, in contrast, are microscopically disordered. It is, and will likely remain, impossible to place the polymers at the wellspecified positions and orientations typical of macroscopic metamaterials. Yet, even these disordered polymeric materials show similarly responsive properties. This raises the question of which of the macroscopic design strategies-material choice, spatial architecture, or mechanical preconditioningmay be implemented in disordered soft materials, and how the anomalous mechanical response prevails in spite of the disorder.

In this paper, we address these questions for control modality (iii): mechanical preconditioning in disordered elastic networks. Our approach is rooted in macroscopic tensegrities: architectures that are geometrically overconstrained (i.e., that possess fewer degrees of freedom than they have constraints) and as a result have one or more so-called states of selfstress (SSS) [26]. These SSS play a key role in determining mechanical response; previous work in physics [27-32] and mechanics [33-36] links their existence to the bulk rigidity of spring networks and granular packings. Even in networks in which the actual self-stress is zero, the mere knowledge of which SSS the network geometrically allows can be used to compute elastic moduli $[29,37]$. But what happens to these moduli, and other mechanical properties, when the actual 
stresses are nonzero? Starting from a network with only one single SSS, a continuum of geometrically indistinguishable network architectures can be defined, differing only in the tension configuration along their bonds. The key question now is whether, and if so how, the geometrically identical networks in such a family of self-stressed states $d o$ differ mechanically. What, in short, is the effect of engaging the SSS in overconstrained networks?

Our paper is laid out as follows. First, we recall the general framework of geometrical mechanics of frames. Then, we detail a method to generate disordered frames with exactly one SSS and no floppy modes. This permits the cleanest discussion of our central question, but it may be generalized to frames possessing multiple SSS. We describe, in general terms, the effect of SSS on the nonaffinity, and along the way we note that, within this model, self-stresses cannot augment mechanical moduli via affine deformation modes. Our disordered SSS frames generally do not deform affinely, and the remainder of our paper details two main effects of engaged prestresses on self-stressed disordered frames: they change the moduli, and at sufficiently high values they can destabilize frames to produce various types of bistability.

\section{GEOMETRICAL MECHANICS: MAXWELL-CALLADINE COUNTING AND STATES OF SELF-STRESS IN FINITE FRAMES}

Following, largely, the conventions of [29], we define a frame to be a spatial distribution of $N_{\mathrm{s}}$ pointlike nodes, connected by $N_{\mathrm{b}}$ bonds. Bonds are either on the boundary or they are internal to the frame. Any initial configuration of the frame in $d$ dimensions is geometrically completely specified by a length- $d N_{\mathrm{s}}$ vector $\mathrm{X}_{0}=\left\{\vec{r}_{0, i}\right\}_{i=1}^{N_{\mathrm{s}}}$ containing all node positions. These initial node positions define the initial lengths $\ell_{0, k}$ of the bonds-if bond $k$ connects nodes $i$ and $j$, then $\ell_{0, k}=$ $\left|\vec{r}_{0, j}-\vec{r}_{0, i}\right|$, and we may collect all these initial lengths into a length- $N_{\mathrm{b}}$ vector $\mathrm{L}_{0}=\left\{\ell_{0, k}\right\}_{k=1}^{N_{\mathrm{b}}}$. Deformed states may now be defined in reference to these initial configurations by specifying the vector of node displacements $\delta \mathrm{X}=\left\{\delta \vec{r}_{i}\right\}_{i=1}^{N_{\mathrm{s}}}$. With any deformation $\delta$ X comes a set of bond length changes $\delta \mathrm{L}=$ $\left\{\delta \ell_{k}\right\}_{k=1}^{N_{\mathrm{b}}}$. To linear order in the bond displacements, $\delta \mathbf{X}$ and $\delta \mathrm{L}$ are related through the $N_{\mathrm{b}} \times d N_{\mathrm{s}}$ compatibility matrix $\mathcal{Q}^{T}$ :

$$
\delta \mathrm{L}=\mathcal{Q}^{T} \delta \mathrm{X} .
$$

In general, each bond $k$ in the frame carries a tension $t_{k}$, directed (for central force networks) along the bond unit vector $\hat{n}_{k}$. The tension is a signed scalar quantity, and in the following we adopt the convention that a positive value of $t_{k}$ corresponds to a tensile force in bond $k$. That is, if $t_{k}$ is positive, then bond $k$ pulls the two nodes it connects toward the middle of bond $k$. Similarly, we may collect all bond tensions into a single length- $N_{\mathrm{b}}$ vector $\mathrm{T}=\left\{t_{k}\right\}_{k=1}^{N_{\mathrm{b}}}$. The tensioned bonds exert forces on the nodes that they connect, which may be computed as the vector sum of all the forces in these bonds. Again, these forces constitute a length- $d N_{\mathrm{s}}$ vector $\mathrm{F}=\left\{\vec{f}_{i}\right\}_{i=1}^{N_{\mathrm{s}}}$. Node forces and the bond tensions inhabit the same geometry that relates node positions and bond extensions, and therefore they are related to each other in a similar fashion:

$$
\mathrm{F}=-\mathcal{Q} \mathrm{T}
$$

with $\mathcal{Q}$ the $d N_{\mathrm{s}} \times N_{\mathrm{b}}$ equilibrium matrix, the transpose of the compatibility matrix. For a finite frame to be at mechanical equilibrium, the net force on each of its nodes must be zero: $\mathrm{F}=0$. Now, the null space of $\mathcal{Q}^{T}$ contains those node displacements $\delta \mathbf{X}$ that do not result in any change in any of the bond lengths; $\delta \mathrm{L}=0$. These displacements (which, in general, involve multiple or even all nodes moving in concerted fashion) are called the zero modes of the frame. All two-dimensional (2D) frames have at least three zero modes; the trivial three correspond to two translations and a single rotation. Zero modes other than these three are called floppy modes in the physics literature, and mechanisms in the mechanical literature. They represent zero-energy deformations of the frame. The dimension of the null space of $\mathcal{Q}^{T}$ is thus the number of independent zero modes.

The null space of $\mathcal{Q}$ contains those tensions T that do not result in any net force on any of the nodes such that $F=0$. Of course, these force balance equations are always trivially solved by $\mathrm{T}=0$, but we will be interested in the nontrivial solutions. Such configurations of nonzero bond tensions, which still give rise to overall mechanical equilibrium, are called states of self-stress (SSS). They may arise for purely topological reasons, or because of special geometries that affect the rank of $\mathcal{Q}$ such as crystalline order or strain-induced rearrangements [26,31,38,39].

The original Maxwell counting argument [40] asserts that the number of zero modes of the frame equals the number of degrees of freedom (here, in $d$ dimensions) minus the number of constraints imposed by the springs (one per bond),

$$
N_{0}=d N_{s}-N_{b} \text {. }
$$

However, since every state of self-stress represents a redundant connection in the system, the number of SSS must be subtracted from the number of bonds, which leads to the modified, Maxwell-Calladine count,

$$
N_{0}=d N_{s}-\left(N_{b}-N_{\mathrm{ss}}\right) \text {. }
$$

Calling $v=\operatorname{dim} \operatorname{ker} \mathcal{Q}^{T}-\operatorname{dim} \operatorname{ker} \mathcal{Q}$ the index of $\mathcal{Q}$ (and noting that, as explained above, $v=N_{0}-N_{\text {ss }}$ ), we obtain the general Maxwell-Calladine "index theorem" [29],

$$
v=d N_{s}-N_{b} \text {. }
$$

We call a frame rigid when it has no zero modes other than the three trivial ones, $N_{0}=3$.

Focusing for a moment on the SSS, let us suppose that a given frame has a single state of self-stress, that is, the dimension of the solution space of the equation $\mathcal{Q} \mathrm{T}=0$ is 1 . We are then free to choose a basis vector $\mathrm{T}_{\mathrm{ss}}=\left(t_{\mathrm{ss}, 1}, t_{\mathrm{ss}, 2}, \ldots, t_{\mathrm{ss}, N_{\mathrm{b}}}\right)$ for this kernel space in any way we like. As soon as we do, a continuous family of possible self-stresses may be defined through a scalar parameter $\alpha$-obviously any vector $\alpha \mathrm{T}_{\text {ss }}$ is also an admissible, equilibrium SSS of the exact same frame but at a different self-stress. This degeneracy is at the heart of our present paper: we will ask how the mechanical properties change as we change the self-stress in geometrically identical frames. To fix the normalization, we choose our reference basis vector such that the actual bond tensions in the network may be written as $t_{k}=\Pi t_{\mathrm{ss}, \mathrm{k}}$, where $\Pi=-\frac{1}{2} \operatorname{Tr}(\sigma)$ (with $\sigma$ the Cauchy stress tensor) is the pressure carried by the boundary bonds of the frame. In practice, this means that 
we normalize $\mathrm{T}_{\mathrm{ss}}$ such that the sum of the four boundary tensions satisfies $t_{\mathrm{ss}, 1}+t_{\mathrm{ss}, 2}+t_{\mathrm{ss}, 3}+t_{\mathrm{ss}, 4}=-2$. This choice also facilitates comparisons between free-standing frames and osmotically swollen periodic frames with the same internal bond geometry and bond forces.

So far, we have discussed the mechanics of frames in entirely geometrical terms. Generally, an elastic "constitutive relation" relates the bond extensions and the bond tensions. In most of the following, we will assume that the tension of a single bond, stretched to a length $\ell_{k}$ and with a rest length $\ell_{\mathrm{eq}, k}$, is given by

$$
t_{k}=\frac{Y}{\ell_{\mathrm{eq}, k}}\left(\ell_{k}-\ell_{\mathrm{eq}, k}\right),
$$

with $Y$ a force scale that, if one imagines constructing the frame out of beams made from a linearly elastic material, can be interpreted as the Young's modulus of that material times the cross-sectional area of the beams. Note the sign convention: a stretched bond has a positive $t_{k}$ and pulls its nodes toward its middle. The above implies that the elastic energy $\varepsilon_{k}$ of a single stretched bond is

$$
\varepsilon_{k}=\frac{Y}{2 \ell_{\mathrm{eq}, k}}\left(\ell_{k}-\ell_{\mathrm{eq}, k}\right)^{2} .
$$

Summing over all bonds and using Eq. (1), this gives the total elastic energy of the frame deformed relative to a forcebalanced reference configuration, in terms of the node displacements $\delta \mathbf{X}$. For small $\delta \mathbf{X}$, this may be written (summing over repeated indices) as

$$
\mathcal{E}(\delta \mathbf{X})=\mathcal{E}(0)+\frac{1}{2} \delta \mathbf{X}_{i}(\mathcal{H})_{i j} \delta \mathbf{X}_{j}^{\top} .
$$

The elements of the stiffness matrix or Hessian $\mathcal{H}$ are then defined by $\mathcal{H}_{i j} \equiv \frac{\partial^{2} \mathcal{E}}{\partial \delta \mathrm{X}_{i} \partial \delta \mathrm{X}_{j}}$ and encode the rigidity of the frame against general deformations. In particular, negative eigenvalues of the Hessian matrix signal mechanical instabilities, since the corresponding eigenvector defines a direction of decreasing energy in Eq. (8); deformations in that direction happen spontaneously. For stress-free initial configurations, $\mathcal{H}$ can be written as $\mathcal{H}=\mathcal{Q K} \mathcal{Q}^{T}$ [37], with $\mathcal{K}$ a diagonal matrix encoding the stiffnesses $Y / \ell_{\mathrm{eq}, k}$, but we will use it in its full form, so that we can use Eq. (8) to establish stability boundaries in prestressed frames.

\section{SELF-STRESSED FRAMES: GENERATION AND MECHANICAL ANALYSIS}

We model inhomogeneously stressed materials using spring networks with explicit boundaries, which we call freestanding frames. In the absence of external preloading, the total stress in these frames is zero, unlike periodic systems in which the fixed shape and size of the periodic unit cell can induce prestress. Self-stresses are therefore the only prestress in these systems, and for general networks the actual selfstress is some nonzero linear combination of the states of self-stress (SSSs) of the system. To keep things tractable, we focus on frames with a single SSS, ranging from Snelson's X, the canonical square with $N_{\mathrm{s}}=5$ nodes connected by $N_{\mathrm{b}}=8$ bonds shown in Fig. 1(a) [41], to more complex networks such as that shown in Fig. 1(c). The entire family of freestanding frames is constructed such that they do not have any

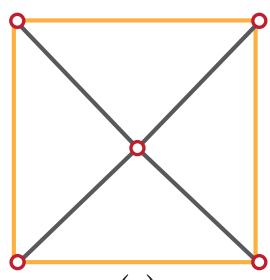

(a)

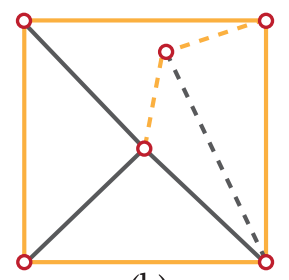

(b)

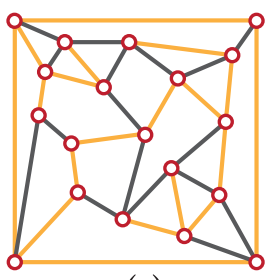

(c)
FIG. 1. (a) A simple network with a single SS state, (b) edge splitting moves on the frame, and (c) an anisotropic frame network with compressed (yellow) and tensile (gray) bonds under freestanding conditions. The stressed bonds represent the state of selfstress.

floppy modes and they obey $2 N_{\mathrm{s}}-N_{\mathrm{b}}=2$, so that according to Eq. (5) they are guaranteed to have exactly one state of self-stress. We set the size of these frames to $1 \times 1$, fixing our unit of length. No other length scales are involved in the definition of the frames, as the average bond length will simply be a function of the number of nodes.

The procedure to generate the more complex frames such that they are still guaranteed to possess exactly one state of self-stress is a repeated application of a modification known as the bond-splitting Henneberg moves [42]. As illustrated in Fig. 1(b), the move amounts to adding a node in an existing bond and connecting this node to another existing node. At the same time, the geometry of the network is kept as generic as possible by displacing the newly added node such that the two halves of the bond it split are no longer collinear. This procedure trivially conserves the difference $N_{0}-N_{\text {sss }}$ because it adds two degrees of freedom as well as two constraints. Furthermore, as long as the three bonds of the newly created node all point in different directions, $N_{0}$ and $N_{\text {sss }}$ are individually conserved. To keep the distribution of bond lengths narrow, we iteratively apply this procedure to the longest bond in the network. Figure 1(c) shows an example of a result of this procedure after 14 iterations. By construction, this frame has one state of self-stress and no floppy modes. The coordination number of the frame after $n$ iterations is $z=2 N_{\mathrm{b}} / N_{\mathrm{s}}=(16+4 n) /(5+n)$ : The average number of bonds per node increases with each iteration and asymptotically approaches the isostatic limit $z=4$. These finite frames serve as a convenient template to conduct our numerical calculations to study the mechanical response.

To isolate the effect of the prestress on the mechanical response, we analyze our frames as spring networks with a fixed geometry, varying the rest lengths $\ell_{\text {eq }}$ of the springs in order to tune the amount of prestress, parametrized by the boundary-bond pressure $\Pi$. The fixed geometry is specified via the positions of the nodes, which set the initial lengths $\ell_{0}$ of the springs. Now, the initial tensions in the frame are no longer zero, and Hooke's law [Eq. (6)] gives

$$
\Pi t_{\mathrm{ss}, i}=\frac{Y}{\ell_{\mathrm{eq}, i}}\left(\ell_{0, i}-\ell_{\mathrm{eq}, i}\right),
$$

from which, for each spring $i$, the rest lengths $\ell_{\mathrm{eq}, i}$ can be determined as a function of prestress $\Pi$. In the rest of the paper, we fix the arbitrary overall force scale by setting $Y=1$. Requiring that Eq. (9) has a positive solution for $\ell_{\mathrm{eq}, i}$ implies 
a constraint $\Pi t_{\mathrm{ss}, i}>-1$, which gives a lower (upper) bound on $\Pi$ via bonds for which $t_{\mathrm{ss}, i}$ is positive (negative). The equilibrium length of bond $i$ will diverge as these bounds are approached. We restrict our analysis of these frames to values of $\Pi$ that fall within the physically accessible range of prestresses,

$$
\Pi_{\min }<\Pi<\Pi_{\max }
$$

with

$$
\Pi_{\min }=\frac{-1}{\max _{i}\left(t_{\mathrm{ss}, i} \mid t_{\mathrm{ss}, i}>0\right)}, \quad \Pi_{\mathrm{max}}=\frac{-1}{\min _{i}\left(t_{\mathrm{ss}, i} \mid t_{\mathrm{ss}, i}<0\right)} .
$$

The total energy stored in the frame now depends on the magnitude of the prestress $\Pi$ via the extension of the springs in the undeformed state $\ell_{0, i}-\ell_{\mathrm{eq}, i}$, and on any subsequent deformations that change the lengths of the springs further by an amount $\delta \ell_{i}$. Summing the harmonic spring energy over all springs, we obtain

$$
\mathcal{E}(\Pi)=\frac{1}{2} \sum_{i=1}^{N_{b}}\left(\frac{1}{\ell_{\mathrm{eq}, i}}\right)\left(\ell_{0, i}+\delta \ell_{i}-\ell_{\mathrm{eq}, i}\right)^{2} .
$$

Thus, $\mathcal{E}(\Pi)$ represents both the work needed to prepare the prestressed frame as well as the work involved in deforming the prestressed frame. Rewriting this energy in such a way that it references only the initial state as specified by the initial lengths $\ell_{0, i}$ and the prestress $\Pi$ using Eq. (9), we write

$$
\begin{aligned}
\mathcal{E}(\Pi) & =\frac{1}{2} \sum_{i=1}^{N_{b}}\left(1+\Pi t_{\mathrm{ss}, i}\right) \ell_{0, i}\left(\frac{\Pi t_{\mathrm{ss}, i}}{1+\Pi t_{\mathrm{ss}, i}}+\frac{\delta \ell_{i}}{\ell_{0, i}}\right)^{2} \\
& \equiv \mathcal{C}(\Pi)+\mathcal{E}(0)+\Delta \mathcal{E}(\Pi)
\end{aligned}
$$

Here,

$$
\mathcal{C}(\Pi)=\frac{1}{2} \sum_{i=1}^{N_{\mathrm{b}}} \frac{\Pi^{2} t_{\mathrm{ss}, i}^{2}}{1+\Pi t_{\mathrm{ss}, i}} \ell_{0, i}
$$

is the constant that measures the work performed on the frame to bring it to the prestressed state (i.e., the shift in the zerostrain energy),

$$
\mathcal{E}(0)=\frac{1}{2} \sum_{i=1}^{N_{b}} \frac{\delta \ell_{i}^{2}}{\ell_{0, i}}
$$

is the deformation energy at zero prestress, and

$$
\Delta \mathcal{E}(\Pi)=\sum_{i=1}^{N_{\mathrm{b}}} \Pi t_{\mathrm{ss}, i}\left(1+\frac{\delta \ell_{i}}{2 \ell_{0, i}}\right) \delta \ell_{i}
$$

is an additional, new prestress-dependent change in the deformation energy. We will be investigating under what conditions a nonzero $\Pi$ changes the mechanical moduli of the frame, and therefore we will consider what happens to each of the three components of the energy when the boundary of the frame is subjected to a deformation. That is, nodes $\vec{r}_{B}$ on the frame boundary are displaced to new positions $\vec{r}_{B}^{\prime}$ according to some deformation gradient tensor $\Lambda$ :

$$
\vec{r}_{B}^{\prime}=\Lambda \cdot \vec{r}_{B}
$$

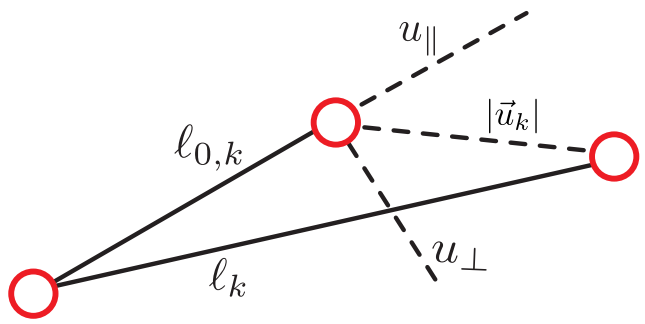

FIG. 2. Decomposing the relative displacement of nodes $i$ and $j$ into components parallel $\left(u_{\|}\right)$and perpendicular $\left(u_{\perp}\right)$ to the original bond vector provides a convenient way to express the change in length $\delta \ell_{k}=\ell_{k}-\ell_{0, k}$.

The interior (nonboundary) nodes in a frame subject to such a deformation will, in general, respond nonaffinely, exploiting their freedom to displace differently from boundary nodes to reduce the incurred bond-stretching energy. For general nodes, therefore, the displacement may be written as

$$
\vec{r}_{i}^{\prime}=\Lambda \cdot \vec{r}_{i}+\vec{\delta}_{i}
$$

With this, the displacement vector $\vec{u}_{k}$ of bond $k$, connecting nodes $i$ and $j$,

$$
\vec{u}_{k}=\left(\vec{r}_{j}^{\prime}-\vec{r}_{i}^{\prime}\right)-\left(\vec{r}_{j}-\vec{r}_{i}\right),
$$

may be expressed in terms of $\Lambda$ and $\hat{n}_{k}$, the unit vector along bond $k$, as

$$
\vec{u}_{k}=\ell_{0, k}\left(\Lambda-\mathbb{1}_{2}\right) \cdot \hat{n}_{k}+\vec{\Delta}_{k}
$$

$\vec{\Delta}_{k}=\vec{\delta}_{j}-\vec{\delta}_{i}$ is the nonaffine part of the bond displacement vector. Now, the change in the length of bond $k$ can be written in terms of the parallel $\left(u_{k, \|}=\hat{n}_{k} \cdot \vec{u}_{k}\right)$ and perpendicular $\left(u_{k, \perp}=\hat{p}_{k} \cdot \vec{u}_{k}\right.$, with $\hat{p}_{k}$ a unit vector perpendicular to $\left.\hat{n}_{k}\right)$ components of the relative displacement of the two nodes (see Fig. 2), as

$$
\begin{aligned}
\delta \ell_{k} & =\left|\left(\vec{r}_{0, j}-\vec{r}_{0, i}\right)+\left(\delta \vec{r}_{j}-\delta \vec{r}_{i}\right)\right|-\ell_{0, k} \\
& =\left|\left(\ell_{0, k}+u_{k, \|}\right) \hat{n}_{k}+u_{k, \perp} \hat{p}_{k}\right|-\ell_{0, k} \\
& =\ell_{0, k}\left(1+2 \ell_{0, k}^{-1} u_{k, \|}+\ell_{0, k}^{-2}\left(u_{k, \|}^{2}+u_{k, \perp}^{2}\right)\right)^{1 / 2}-\ell_{0, k} .
\end{aligned}
$$

To access the moduli, we must now ask how each of the three terms in the energy of the prestressed frame, Eq. (13), is affected by the deformation $\Lambda$. The first term, $\mathcal{C}(\Pi)$, is unaffected as it does not depend on the deformation. The second term, $\mathcal{E}(0)$, does depend on the deformation but not directly on the prestress (we come back to this in a moment). The third term encodes the direct coupling between prestress and deformation and, using Eq. (16), reduces to

$$
\Delta \mathcal{E}(\Pi)=\sum_{k=1}^{N_{\mathrm{b}}} \Pi t_{\mathrm{ss}, k} u_{k, \|}+\frac{1}{2} \sum_{k=1}^{N_{\mathrm{b}}}\left(\frac{\Pi t_{\mathrm{ss}, k}}{\ell_{0, k}}\right)\left(u_{k, \|}^{2}+u_{k, \perp}^{2}\right) .
$$

Note that Eq. (21) is not an expansion in $u$. Due to the specific structure of Eq. (16), the square roots drop out and there are no terms beyond those quadratic in $u$.

A logical place to start the analysis is to consider an affine deformation, one in which $\vec{\Delta}_{k}=0$ for all bonds. In that case, every point $\vec{r}$ in the reference frame is mapped onto its 
deformed image $\vec{r}^{\prime}$ according to just the deformation gradient tensor $\Lambda: \vec{r}^{\prime}=\Lambda \cdot \vec{r}$. In Appendix, we show that in this particular case something curious happens: both displacementdependent terms in Eq. (21) become proportional to the overall stress tensor $\sigma_{\alpha \beta}$. In particular, the linear term can be written as

$$
\sum_{k=1}^{N_{b}} t_{s s, k} u_{k, \|} \propto\left(\Lambda-\mathbb{1}_{2}\right)_{\alpha \beta} \sigma_{\alpha \beta},
$$

and the quadratic term as

$$
\sum_{k=1}^{N_{b}}\left(\frac{t_{s s, k}}{\ell_{0, k}}\right)\left(u_{k, \|}^{2}+u_{k, \perp}^{2}\right) \propto\left(\Lambda-\mathbb{1}_{2}\right)_{\mu \alpha}\left(\Lambda-\mathbb{1}_{2}\right)_{\mu \beta} \sigma_{\alpha \beta} .
$$

In free-standing frames, the total stress tensor $\sigma_{\alpha \beta}$ is zero because there are no external forces. This brings us to a first conclusion about the effect of prestress: in an affine system,

$$
\mathcal{E}(\Pi)=\mathcal{C}(\Pi)+\mathcal{E}(0)
$$

and since the energy shift $\mathcal{C}(\Pi)$ does not depend on the deformation, all derivatives with respect to the deformation, including those that yield the moduli, are unchanged. In short, engaging the state of self-stress by applying a prestress to the frame can never change the mechanical moduli of an affinely deforming frame in the case in which the extensional moduli of the bond springs vary inversely with bond length. While these conditions may appear restrictive, we note that the class of systems to which they apply includes all crystalline frames with one node per unit cell. In such systems, nodes are constrained by local symmetries to move affinely. For the large class of these systems that also have a single bond length, the scaling of the spring constant with the equilibrium length is immaterial. All such systems are forbidden from having prestress-dependent moduli. We suggestively attribute the fact that this surprising result has not been reported before to the usual focus on periodic systems, rather than our free-standing frames. We will address the connection between our findings and the tensegrity literature in the discussion.

Fortunately, there is also a large class of nonaffinely deforming frames to which the above restriction does not apply. Do these have prestress-dependent moduli? The answer to this question is yes, and to see how it arises we now assume that $\vec{\Delta}_{k} \neq 0$ for some, or all, of the bonds of the frame. Doing so again leaves $\mathcal{C}(\Pi)$ unaffected, as it does not depend on the deformation. The other two terms $d o$ change. We may split the total energy into an affine (A) part (i.e., independent of $\vec{\Delta}_{k}$ ) and two new nonaffine (NA) parts as

$$
\mathcal{E}(\Pi)=\mathcal{E}^{\mathrm{A}}(\Pi)+\mathcal{E}^{\mathrm{NA}}(0)+\Delta \mathcal{E}^{\mathrm{NA}}(\Pi),
$$

where we recognize the part of the nonaffine deformation energy correction that does not explicitly depend on the prestress,

$$
\begin{aligned}
\mathcal{E}^{\mathrm{NA}}(0)= & \frac{1}{2} \sum_{k=1}^{N_{b}} \ell_{0, k}\left[\left(\left|\Lambda \cdot \hat{n}_{k}\right|^{2}+\Gamma_{k}\right)^{1 / 2}-1\right]^{2} \\
& -\frac{1}{2} \sum_{k=1}^{N_{b}} \ell_{0, k}\left[\left|\Lambda \cdot \hat{n}_{k}\right|-1\right]^{2},
\end{aligned}
$$

and another part that couples the prestress and the nonaffinity

$$
\Delta \mathcal{E}^{\mathrm{NA}}(\Pi)=\frac{1}{2} \sum_{k=1}^{N_{b}} \ell_{0, k} \Pi t_{\mathrm{ss}, k} \Gamma_{k} .
$$

Notation has been condensed somewhat by introducing

$$
\Gamma_{k} \equiv \frac{2}{\ell_{0, k}}\left(\Lambda \cdot \hat{n}_{k}\right) \cdot \vec{\Delta}_{k}+\frac{1}{\ell_{0, k}^{2}}\left|\vec{\Delta}_{k}\right|^{2} .
$$

Clearly, the affine limit $\vec{\Delta}_{k}=0$ corresponds to $\Gamma_{k}=0$ in which both NA energy terms are zero. While it might appear that $\mathcal{E}^{\mathrm{NA}}(0)$ does not depend on the prestress since $\Pi$ does not appear, in fact it does. The reason for this is that the ground-state energy of the nonaffinely deforming frame is defined as the minimum of Eq. (25) over the nonaffine node displacements $\vec{\delta}_{i}$. Through the coupling term Eq. (27), this minimum will be attained at different values of $\Gamma_{k}$, which in turn affects the value of the nonaffine deformation energy Eq. (26).

While the mathematical conditions for this minimization may be written down analytically, due to the combination of vectorial (terms proportional to the orientation of $\vec{\Delta}_{k}$, particularly in relation to the unit bond vector $\hat{n}_{k}$ ) and scalar (terms proportional to $\left|\vec{\Delta}_{k}\right|$ ) aspects, solving them in the general case is, in practice, possible only numerically. A further complication is that the SSS components $t_{\mathrm{ss}, k}$ may be both positive and negative, and indeed both signs generally occur within a single SSS. As a result, there is no straightforward way to read off the high- or low prestress limits. In the special case, however, where all internal prestresses are extensional (that is, all $t_{\mathrm{ss}, k}$ for internal bonds are positive), we conclude that the nonaffine energy terms favor $\vec{\Delta}_{k}$ 's that are small and/or perpendicular to the bond unit vector (both of which contribute to the minimization of $\Gamma_{k}$, which dominates the nonaffinity-prestress coupling term in the energy [Eq. (27)] at high $\Pi$ ). Based on this, we can conclude that for purely extensional SSS's, large prestresses will suppress nonaffinity, which should manifest in increasingly small values of $\left|\vec{\Delta}_{k}\right|^{2}$ at higher $\Pi$.

In general, and as we will detail in the following, prestresses due to engaged states of self-stress can and do affect the moduli of nonaffinely deforming frames. Since the affine deformation energy represents an upper bound to the actual (nonaffine) energy (affinity is a constraint, the release of which will lead the system down, not up, in the energy landscape), the linear modulus of a generic frame must be lower than that of the same frame deforming affinely. In the following, we show that changing the prestress $\Pi$ allows for tuning of the moduli up to their affine values, and that the mechanism by which this happens is the suppression of nonaffine displacements by the prestress. 


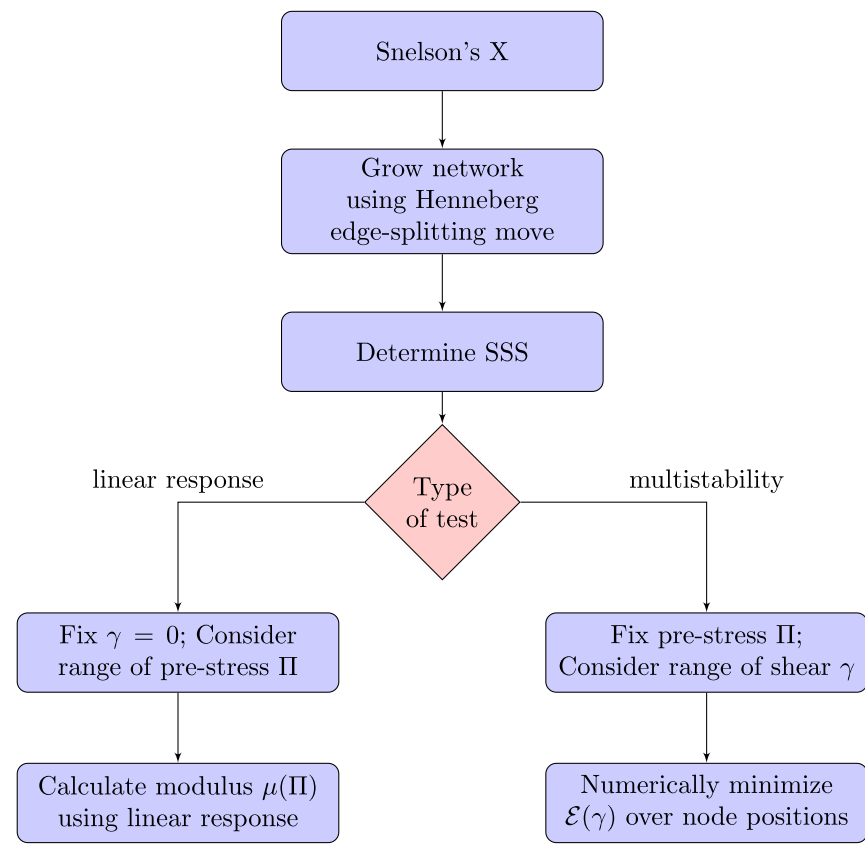

FIG. 3. The flowchart outlines the steps involved in the results presented in Secs. IV (linear response) and V (multistability). The energy of the spring-bead network $\mathcal{E}(\gamma)$ is numerically minimized using the quasi-Netwon method on MATHEMATICA (version 11.0). The linear-response methods are described in Ref. [43].

Having shown that an affinely deforming frame is insensitive to the magnitude of prestresses in the network, we now analyze systems with nonaffine deformations. We characterize these using the differential (linearized) shear modulus. Our main results are that (i) the differential shear modulus does depend on the prestress, (ii) this happens via a generally repressive coupling between the nonaffine displacements and the prestress, and (iii) prestress can induce instabilities in the frame, a fact most readily observed by noting that there are values of $\Pi$ at which the shear modulus becomes negative. In Sec. IV we detail findings (i) and (ii), turning to finding (iii) in Sec. V.

\section{LINEAR RESPONSE OF SELF-STRESSED FRAMES: MODULUS AND NONAFFINITY}

Our procedure for analyzing response, both linear and nonlinear, is summarized in the flowchart in Fig. 3. First, we generate a frame applying iterated bond-splitting Henneberg moves to Snelson's X as described in Sec. III. Then, we dial in a value for prestress $\Pi$; this leaves the geometry of the frame unaltered but is reflected in a set of bond equilibrium lengths $\ell_{\text {eq }, i}$. In those cases in which we want frames with exclusively tensile forces on the internal bonds, we temporarily set the rest length to zero and allow the internal bonds to relax, keeping the boundary fixed. The state that remains is guaranteed to have only extended bonds internally; we then reset the initial lengths $\ell_{0, k}$ to their relaxed values, after which we can use the procedure detailed around Eq. (9) to set the self-stress $\Pi$ by varying the equilibrium lengths $\ell_{\mathrm{eq}}$.

For the results in this section dealing with the linear response, we vary the prestress $\Pi$, extracting the shear modulus
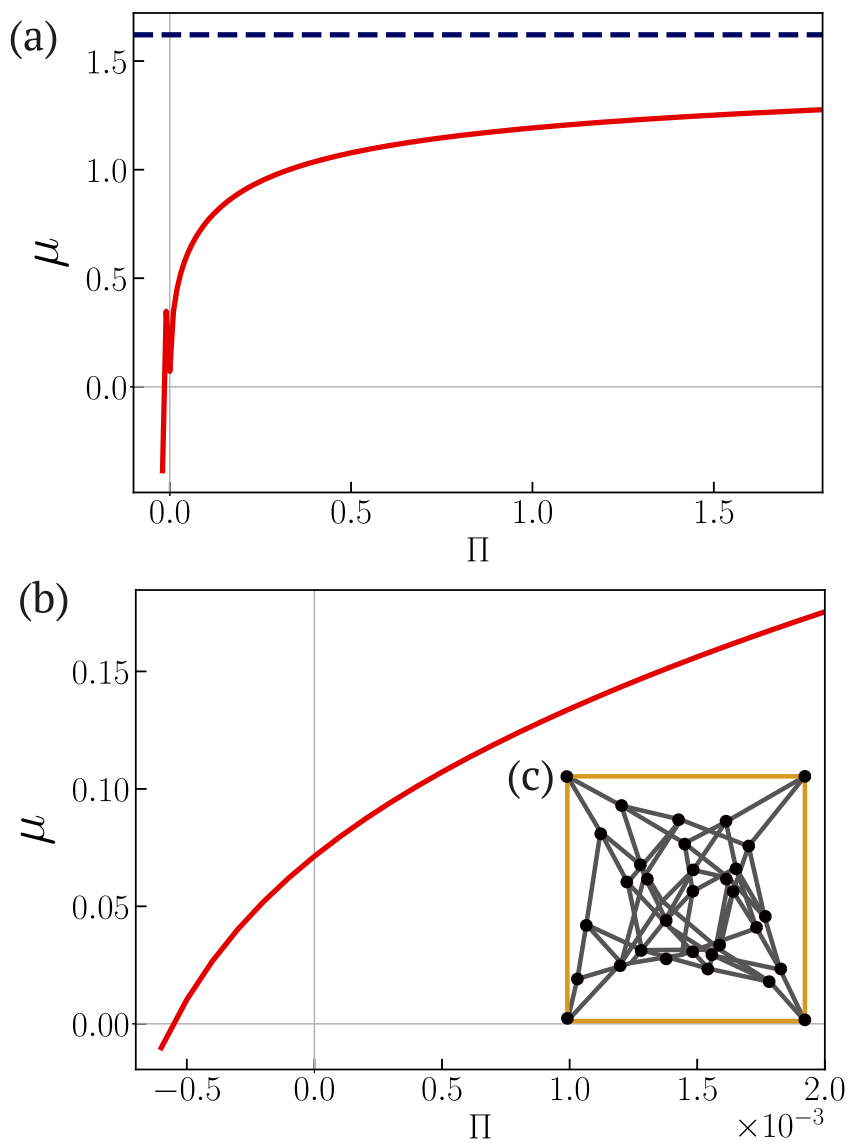

FIG. 4. (a) Prestress dependence of the shear modulus $\mu$ of a nonaffinely deforming free-standing frame [shown in (c)]. As the self-stress $\Pi$ is increased, the shear modulus (red) of geometrically indistinguishable frames changes, and it is bounded by the affine shear modulus $\mu_{\text {aff }}$ (dashed blue line). For this particular frame, $\Pi_{\min }=-2.52, \Pi_{\max }=1.82$, and $\mu_{\mathrm{aff}}=1.62$. (b) Zoom-in of the region near $\Pi=0$, showing that the modulus becomes negative for $\Pi<0$, signaling that the frame becomes unstable to simple shear. (c) Frame with 64 tensile (gray) and 4 compressive (yellow) bonds connecting across 35 nodes.

of the frame from an expansion of the total spring energy to second order in strain $\gamma$, as described in Ref. [43]. In the next section, dealing with nonlinear response, we will deform the frames by imposing a displacement to the four corners. This deformation is prescribed by the deformation gradient tensor $\Lambda$, which is generally parametrized by some scalar measure of the strain. We then let the positions of all noncorner nodes relax (which amounts to exploring the space of nonaffine node displacements $\vec{\delta}_{i}$ ) until a minimum of the energy Eq. (25) is reached.

We will mostly be considering the case of simple shear, for which the boundary points are displaced according to the deformation gradient tensor,

$$
\Lambda(\gamma)=\left[\begin{array}{ll}
1 & \gamma \\
0 & 1
\end{array}\right] .
$$

In this case, the scalar strain measure is the shear strain $\gamma$. Our main finding is that a nonzero value of $\Pi$ does indeed augment the shear modulus $\mu$. Figure 4 illustrates this point. 
The linear shear modulus is seen to vary with the self-stress over the range of physically relevant self-stresses (i.e., those corresponding to positive equilibrium lengths). Throughout this regime, two behaviors stand out: First, in the mechanically stable regime $(\mu>0)$, the shear modulus changes with $\Pi$, and secondly, self-stress can induce mechanically unstable $(\mu<0)$ states. This response is generic; although the values of $\Pi_{\min }$ and $\Pi_{\max }$ differ from frame to frame, we see the same behavior in all frames we have analyzed. This then is finding (i): as opposed to affinely deforming frames where there can be no effect, self-stress controls the linear mechanical response in generic, nonaffinely deforming frames.

Digging down into the nature of this dependency, we focus first on linear response in the mechanically stable regime. The mechanism by which self-stress controls the linear modulus is laid out in Sec. III: the set of $\left\{\vec{\delta}_{i}\right\}_{i=1}^{N_{b}}$ at which the minimum of energy Eq. (25) is attained given a deformation $\Lambda(\gamma)$ depends, through the coupling term Eq. (27), on the prestress.

As detailed in Sec. III, the shear modulus of a nonaffinely deforming prestressed frame can never exceed the shear modulus of the same frame deforming affinely, which in turn is equal to the shear modulus of the non-prestressed frame deforming affinely. We therefore have $\mu \leqslant \mu_{\text {aff }}$.

From Fig. 4, it appears that for higher prestresses the shear modulus approaches its affine limit. This could happen for a variety of reasons, but the most obvious one is that the nonaffine displacements of the nodes $\left\{\vec{\delta}_{i}\right\}_{i=1}^{N_{s}}$ themselves tend to zero. In Sec. III, we demonstrate this to be the predicted dependence for frames possessing only tensile self-stresses on the nonboundary bonds. To verify this prediction we measure the nonaffinity, defined as

$$
N(\gamma, \Pi) \equiv \frac{1}{\gamma^{2}}\left\langle\left(\frac{\left|\vec{\Delta}_{k}\right|}{\ell_{0, k}}\right)^{2}\right\rangle,
$$

with the average $\langle\cdot\rangle$ running over all $N_{b}$ springs. Because $N$ becomes very large as $\Pi \rightarrow 0$, we plot $1 / N$ in Fig. 5 for both simple shear and pure shear deformations. Indeed, increasing $\Pi$, but keeping it below its maximal value, suppresses the magnitude of the nonaffine displacements. By extension, the shear modulus should approach its affine limit.

The nonaffinity measure $N(\gamma, \Pi)$ signals other interesting behaviors. First, it becomes very large (albeit finite, as it should be in a finite-size system) as the system approaches a mechanical instability, marked by the shear modulus becoming negative. In Sec. V we investigate this regime further. For now, we note that apparently large deviations from affine occur at such a point; these correspond to the system acquiring novel state(s) of mechanical equilibrium, possibly quite far away from the reference configuration. This finding echoes the signaling quality of the nonaffinity also observed in [44]. There, too, a cusp in a quantity directly proportional to our $N(\gamma, \Pi)$ marks the floppy-to-rigid transitions occurring in both flexibly hinged and bond-bending fiber networks with periodic boundary conditions.

In case all internal forces have the same sign, we find that the frame becomes unstable if we make all internal bonds carry compressive loads, in accordance with the known destabilizing nature of compressive forces [45-48]. Furthermore, our finding that the deformation of the frame becomes

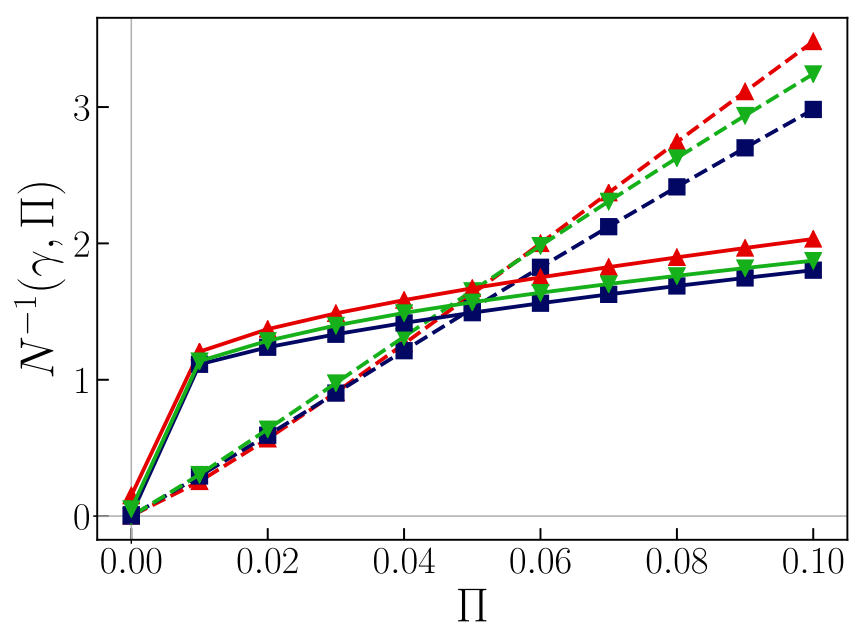

FIG. 5. Prestress dependence of $N^{-1}(\gamma, \Pi)$ for nonaffinely deforming self-standing frames, averaged over an ensemble of 10 network realizations each for 40 nodes (red $\mathbf{\Delta}$ ), 50 nodes (dark blue $\mathbf{\square}$ ), and 60 nodes (green $\boldsymbol{\nabla}$ ). As the self-stress $\Pi$ is increased, the nonaffinity drops, approaching zero corresponding to the affine limit. The closeness of the curves emphasizes that the system size dependence is small. Dashed lines represent the case of simple shear, for which $N \sim 1 / \Pi$ appears to describe the data well over this range of self-stress values [although it should be noted that $N(0)$ is actually finite]. Solid lines describe the nonaffinity for pure shear. Uniform compression shows similar behavior to pure shear (not shown).

increasingly affine as we ramp up the self-stress confirms earlier reports that-for various different systems both in $2 \mathrm{D}$ and 3D-tensile prestresses increase affinity $[28,49,50]$. We do note, however, that conflicting reports also exist that appear to show the nonaffinity rising with prestress. Based on what we are able to conclude here, we cannot rule out that certain systems are more compression-dominated, in which case the interdependence between nonaffinity and prestress could well be oppositely signed.

The fact that tensile prestresses suppress nonaffinity is not unique to shear deformation. When we subject our frames to pure shear, effected by using

$$
\Lambda\left(\gamma_{P}\right)=\left[\begin{array}{cc}
1+\gamma_{P} & 0 \\
0 & 1-\gamma_{P}
\end{array}\right]
$$

and uniform compression, for which $(\epsilon>0)$

$$
\Lambda(\epsilon)=\left[\begin{array}{cc}
1-\epsilon & 0 \\
0 & 1-\epsilon
\end{array}\right],
$$

we see the same behavior. Figure 5 shows this for simple and pure shear; in each of these cases the nonaffinity drops as $\Pi$ is increased, indicating that for general deformations and in frames with purely tensile SSS's, large positive self-stresses suppress nonaffinity and steer the associated moduli to their affine values. Interestingly, this effect appears strongest (in the sense that the range of prestresses compatible with stability is smallest) for simple shear, but regardless: Since any deformation in 2D may be decomposed into shear and extensional components, this establishes finding (ii): a coupling between the prestress and the nonaffinity controls the $\Pi$-dependence of $\mu$. Figure 6 shows that this coupling is also manifested in 

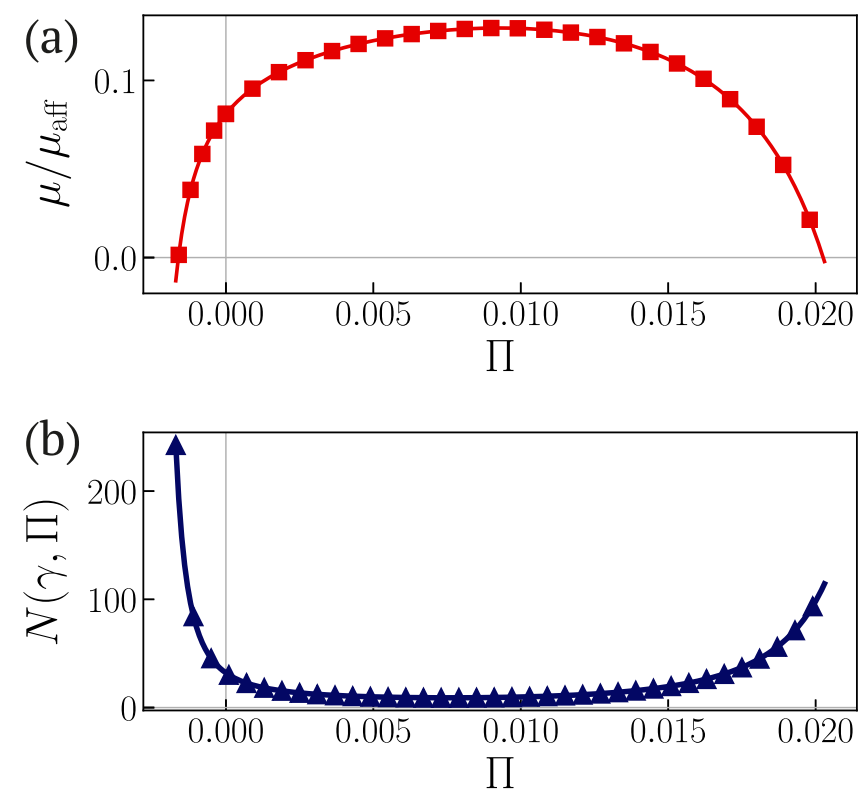

FIG. 6. (a) Prestress dependence of the simple shear modulus of a frame whose SSS contains both tensile and compressive bonds. In such frames, the range of prestresses compatible with mechanical stability is bounded both from below and above. (b) As in the purely tensile frames, the nonaffinity measure signals the instability, blowing up at the stability edges.

frames with a mixed SSS (that is, a SSS in which some bonds are compressed and others are tensed) and that, completely analogous to what happens in purely tensile frames, a steeply increasing nonaffinity foreshadows mechanical instability.

\section{NONLINEAR RESPONSE OF SELF-STRESSED FRAMES: MULTISTABILITY}

We now turn to the behavior of frames whose reference geometry has been rendered unstable by sufficiently large (or small) prestress. Their instability is marked by a negative linear modulus. It is important to note that these instabilities generally do not occur at values of the self-stress corresponding to $\Pi_{\min }$ or $\Pi_{\max }$. We will return to how the instability thresholds may be computed in a moment.

For an unstable network, a negative shear modulus means that the expansion of the energy in the strain variable (which we will assume to be the shear strain $\gamma$ for now) starts out as

$$
\mathcal{E}(\gamma, \Pi) \approx \mathcal{E}(0, \Pi)-\frac{1}{2}|\mu(\Pi)| \gamma^{2}+O\left(\gamma^{3}\right) .
$$

This energy will prompt a spontaneous shear strain $\gamma$, adjusting the shape of the box (and, as this changes, the positions of the nodes) until a new, stable geometry is reached. Because the frame at $\gamma=0$ is in mechanical equilibrium (albeit an unstable equilibrium), there is no linear term in $\gamma$. Generally, networks generated using our Henneberg construction protocol have both tensile and compressive elements in the single state of self-stress spanning the network. As we have seen in the previous paragraph, this means the original square shape of the frame will become unstable if the prestress becomes too high in either direction.

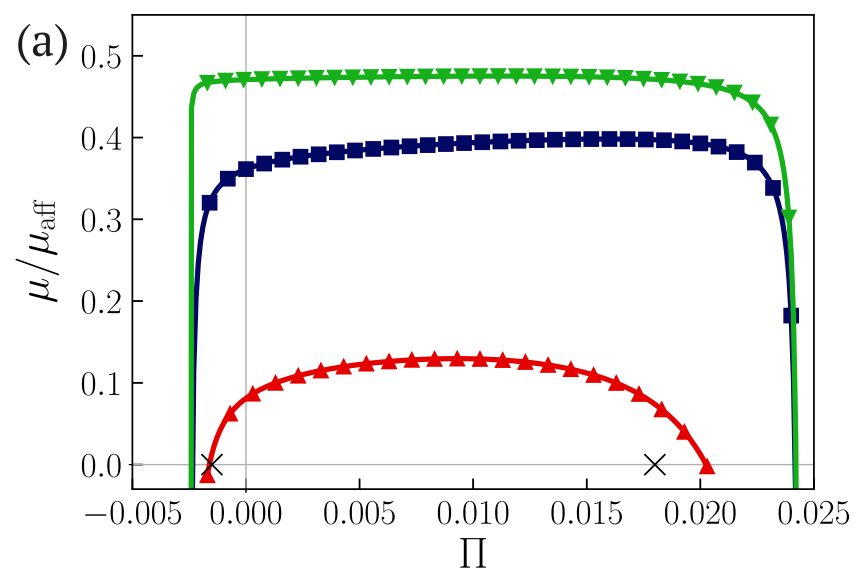

(b)

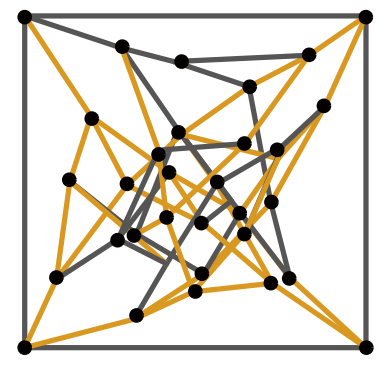

FIG. 7. (a) The simple shear (red $\mathbf{\Delta}$ ), bulk (green $\boldsymbol{\nabla}$ ), and pure shear (dark blue $\mathbf{\square}$ ) modulus (normalized by the affine value $\mu_{\text {aff }}$ ) are shown for $-0.0025<\Pi<0.025$. (b) The corresponding frame has both tensile and compressed bonds, so that both positive and negative $\Pi$ will drive the system to be unstable. The black crosses on the $x$-axis indicate the values of $\Pi$ at which the smallest eigenvalue of the Hessian becomes negative.

Identifying these instabilities via specific moduli gives the values of prestress for which deformation along the oneparameter axis corresponding to that modulus becomes unstable. Within the network, these instabilities can be seen earlier by looking at the lowest eigenvalue of the Hessian matrix of the total spring energy, which becomes negative as soon as one mode becomes unstable. Note that a single unstable mode is not guaranteed to lead to instabilities in the moduli, as the displacements of the boundary points according to that mode generally do not match the imposed deformation gradient tensor $\Lambda$.

As is seen in Fig. 7, we find that destabilizing the system tends to happen at the lowest prestress for simple shear; to explore the consequences of destabilization, we continue to focus on simple shear from hereon.

Once the system has become unstable to simple shear, allowing the system to find its preferred shape along the shear deformation direction should then reveal new stable states that have a nonsquare shape. We have studied the corresponding energy landscape as a function of shear strain $\gamma$ and found that in many cases there was more than one nonsquare stable shape. Thus, our model system can be tuned from monostable (and square) to bistable (switchable between two different shapes) by varying the internal prestress while keeping the initial geometry identical.

We find two distinct types of bistability. Most common is the emergence of two branches in the energy function, with 

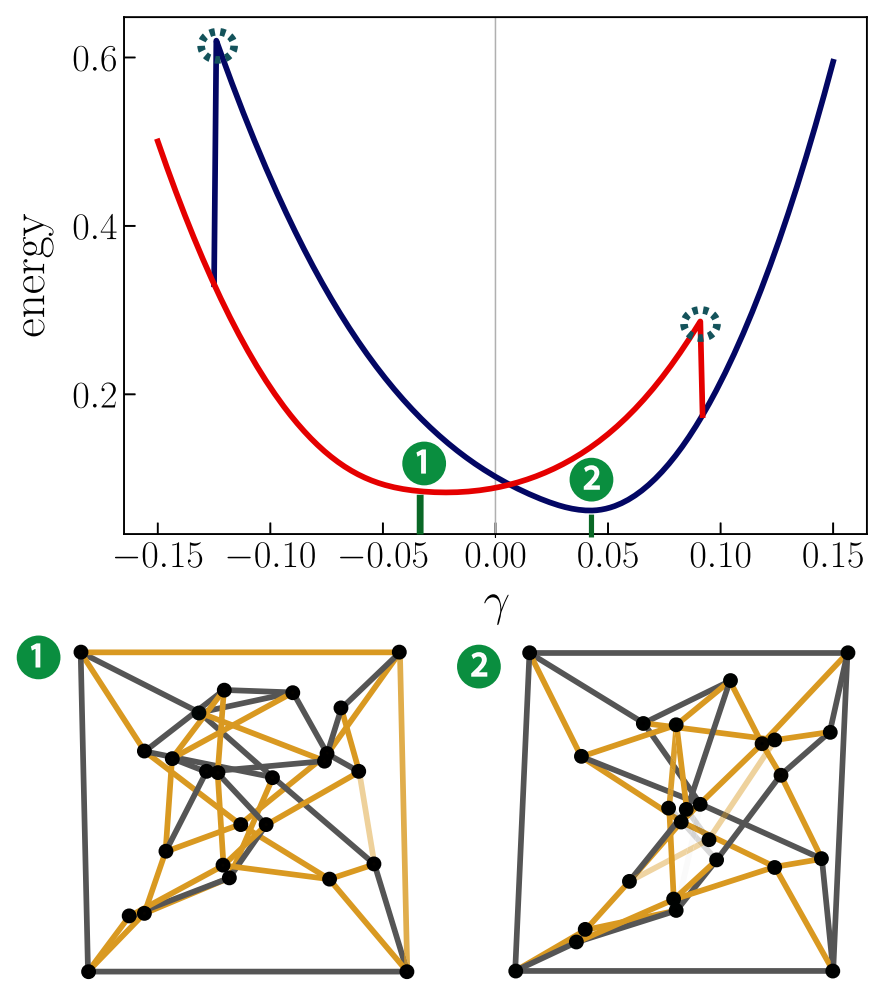

FIG. 8. Energy as a function of shear strain for a bistable frame with hysteresis. Only the two branches of the limit cycle are shown, with the points at which the frame jumps to the lower branch indicated. The frame geometries at the bottom correspond to the two minima labeled by (1) and (2).

discontinuous jumps between them and hysteresis over the course of a strain cycle, as seen in Fig. 8. More rarely, in about $3 \%$ of the frames studied, we see unstable behavior where the energy remains smooth as a function of strain, and where the initially stable state at zero shear is destabilized at finite $\Pi$ giving way to two local minima (see Fig. 9).

The self-stressed frames have a positive shear modulus at $\Pi=0$, as expected since the elastic energy $\mathcal{E}$ is nonnegative and the energy of the reference configuration is $\mathcal{E}(0,0)=0$ (plus our frames have no floppy modes). The frames are subjected to multiple cycles of simple shear until their energy curve attains a steady state. The energy curve is calculated through numerical minimization. There is no noticeable unstable behavior at $\Pi=0$, even though some frames can change their energy path after the first cycle, but the change is smooth. Multistability is most pronounced at nonzero values of prestress.

One such instability is noticed when the prestress is set to values at which the linear shear modulus is negative. At these values, the energy drops instantly at the first shear step, prompting rearrangement of the network, as can be seen in Figs. 9 and 10. Figure 8 also shows that as these frames are sheared, they undergo snap changes in the geometry at strains where the energy jumps between branches corresponding to different shear cycles. The change in distribution of stresses at each strain step makes such hysteretic jumps between energy branches very common in these finite frames. The drop in energy is typically paired with a significant spatial

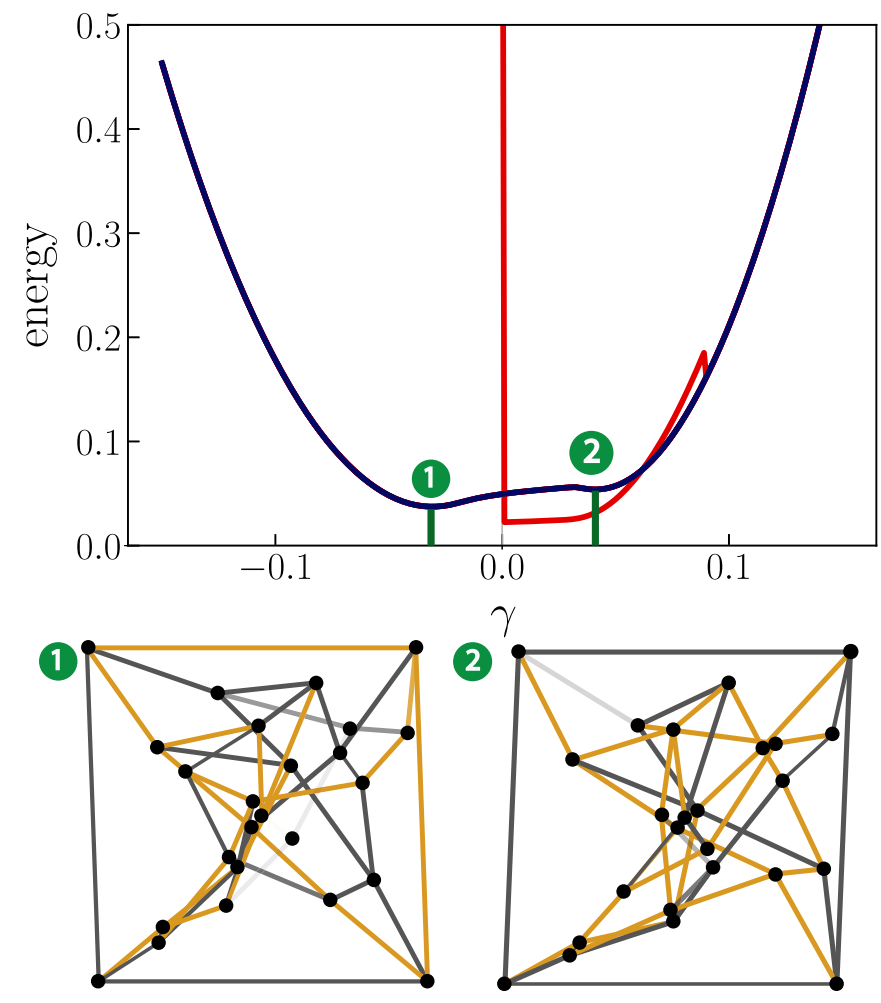

FIG. 9. Energy vs shear strain $\gamma$ for $\Pi=0.009$. The first shear cycle (red) is marked with an immediate drop in the energy, followed by a small jump to a different branch at roughly $10 \%$ strain. The rest of the first and second (blue) shear cycle continues on this branch, which displays two local energy minima at $\gamma=-0.036$ and 0.041 , as illustrated in the graph marked by the green labels. The geometries of the frame in these local energy minima are shown below. The tensile bonds are shown in gray while the compressed bonds are in yellow, and the opacity of the bond is proportional to the tension $t_{\mathrm{ss}, \mathrm{k}}$ of each bond.

rearrangement of the nodes and a decrease in the number of compressed bonds. In some networks, we observed a phenomenon reminiscent of strain hardening: they showed a larger shear modulus in the second shear cycle than in the first.

The hysteretic self-stressed frames thus constitute a mechanical memory device: The overall shape of the frame is indicative of the direction in which the frame was sheared last, and in some cases (in addition to looking at the shape) this information can also be read out by probing the modulus. A closely related behavior is shown in Fig. 10: This frame is unstable in its initial configuration, snaps immediately to a metastable branch, but ultimately goes through another instability, after which it becomes fully stabilized in a new, nonsquare shape. This particular frame can hence report whether or not it has ever been sheared to the left by more than a certain amount (in this example, 7\%), serving as another type of mechanical sensor.

\section{DISCUSSION}

We have studied the effects of self-stresses on the linear and nonlinear mechanical properties of free-standing spring networks or frames. By keeping the geometry constant and 

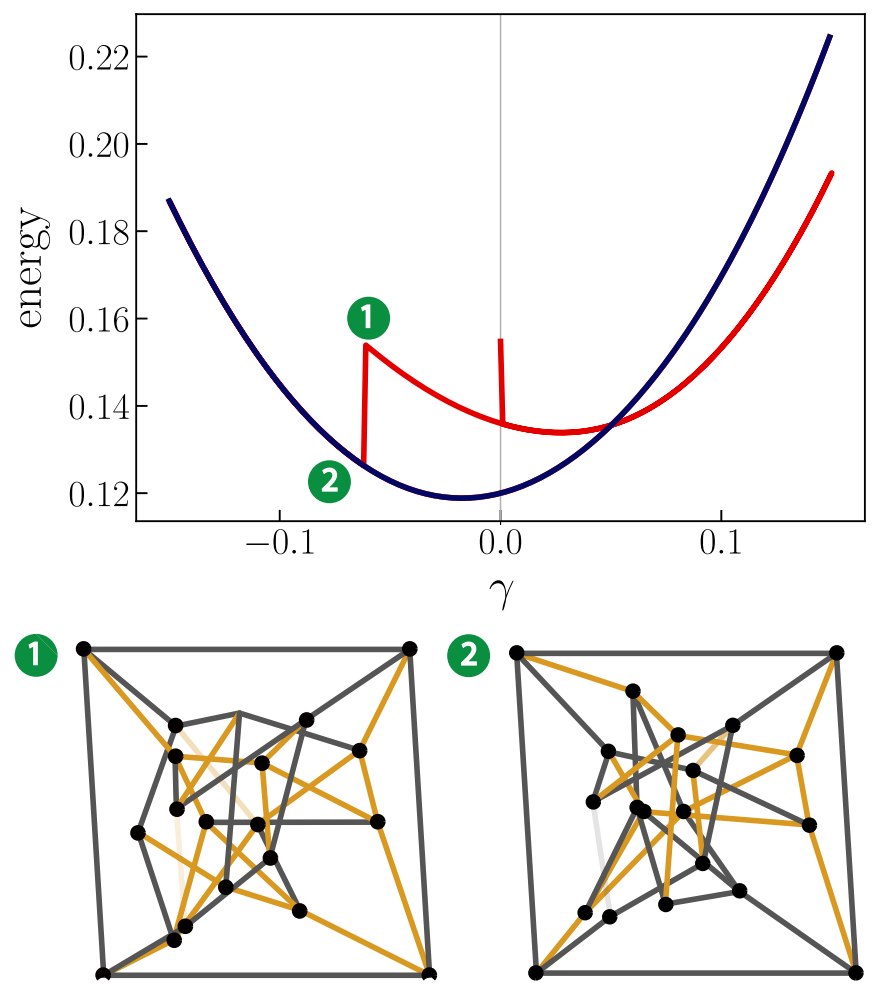

FIG. 10. The energy of the shear deformation is plotted at every strain step $\gamma$ plotted for each cycle. The first cycle is represented in red and the second is represented in dark blue. Note that the following shear cycles follow the blue curve, hence they are not plotted. At $\Pi=0.02$ and $\gamma=-0.062$ the frame makes a hysteretic jump to the blue branch and continues to follow that path for all the negative values of $\gamma$. Interestingly, for $\gamma>0$ the blue curve has greater curvature than the red cycle, indicating an increase in the shear modulus. The local distribution of stresses in the frame is highly heterogeneous, and the opacity of the bonds is scaled to the amount of stresses borne by them.

adjusting the self-stress via the rest lengths of the springs, we isolated the direct effects of the stresses from those mediated by the changes in frame geometry. Within the scope of our model, the energy involved in affine deformations of the frames is independent of the self-stress, so that the moduli can only change if the deformations are nonaffine. We have shown analytically and numerically that in frames in which the internal bonds are all tensile, the self-stress suppresses nonaffinity.

In the context of tensegrities or free-standing frames, it was already known that engaging the self-stress cannot lift a floppy mode to become rigid [36]. This result does not apply to our frames, since these, by construction, have a nonzero shear modulus at $\Pi=0$. Comparing this to our general finding, neither result is more general than the other: Our result, while being applicable to frames that are already rigid at zero self-stress, does rely on the interactions being harmonic springs with a stiffness that is inversely proportional to their equilibrium length.

The extent to which frames do deform affinely is influenced by many factors. First, there may be symmetries in the system that dictate affine deformations. Second, it has been known for decades that networks of springs with zero rest length (such as Gaussian chain networks, an idealized model for rubbers) deform affinely regardless of the prestress and the distribution of stiffnesses [51]. Third, we know that networks that are more densely connected, with many more bonds per node than the isostatic condition demands, typically deform more affinely [43,44,52]. And finally, even systems that do not satisfy any of the above criteria can be made to deform more affinely by way of tensile prestresses. This feature-one of the central findings of this work-is in line with the general stabilizing tendency of tensile prestress noted in [45]. While the effect was observed in simulations of a unit-cell model for polymer networks [49], it does not appear to have been explicitly demonstrated, verified, or derived for many other systems, although many studies on nonaffinity in disordered networks are, retrospectively, consistent with our findings $[28,44,50,53]$. Our findings suggest that it is not strain itself, but rather the tensions incurred as a system is strained, that suppress nonaffinity in these earlier works. Selfstressed frames - tensed but otherwise undeformed systemsallow us to separate the two, proving our point.

Here, we showed that frames with compressive or mixed SSS may become unstable upon increasing the self-stress, and that the approach of the instability is marked by both a decreasing modulus and increasing (possibly diverging) nonaffinity, while purely tensile self-stresses generally suppress nonaffinity.

Going beyond linear properties, we showed that the destabilizing nature of self-stresses leads to frames with interesting energy-strain profiles. These feature strain-induced rearrangements, corresponding to jumps between branches. Some of these show multiple stable minima, with hysteretic behavior between them; others are initially metastable and snap to a single stable minimum after a sufficiently large shear deformation. We speculate that such hysteretic cycles can be used to design self-stress controlled mechanical switches that remember (store) the direction in which they were last sheared. Likewise, the metastable case can serve as a sensor, reporting whether an object has previously been deformed by more than a certain amount. This brings new uses for self-stress-controlled nonlinear mechanical properties to mechanical metamaterials [54].

The multistability of the frames depends on both the geometry and the prestress. As we have no means to construct a bistable frame in a designed way, we used a simple sweep of parameter space to find them. While the precise energy versus strain curve is sensitive to errors in geometry and prestress, we found that, for any given geometry, small errors in the choice of the prestress do not result in any significant deviations.

Experimental realizations of structures with this sort of multistability could be made with the macroscopic force carrying elements typically used for tensegrities. For smallerscale realizations, one could employ 3D-printed cellulosepatterned hydrogel composites [55]. In this case, the desired geometry and inhomogeneous stress distribution would be obtained by swelling a specifically designed 3D-printed object in water. Similarly, we expect that self-stress effects can enhance the already spectacular mechanical properties of double and triple network elastomers [21]. An effective way to harness the benefits of self-stresses would be to introduce elements into 
these materials that are better able to carry compressive loads, such as semiflexible polymers [56], or to explore strategies that build up compressive loads automatically [57].

Unlike the frames we study here, larger systems will likely have many states of self-stress. Preliminary calculations have shown that the second and subsequent SSS(s) have a decreasing contribution to the mechanical response of the network. More work is required to assess the differences between localized states of self-stress, which may be used for patterning materials, and those that are system-spanning, and likely have a more direct effect on the macroscopic mechanical properties.

In summary, and returning to issue (iii) as defined in the Introduction, we have shown that mechanical preconditioning, by means of states of self-stress, represents a powerful control modality able to tune the linear and nonlinear mechanical response of disordered network materials. Designer matter, both macroscopic and molecular, has only just begun to exploit this design principle, which we suspect may be far more broadly leveraged to create new adaptive materials with a tailored response.

\section{ACKNOWLEDGMENTS}

We are grateful to Thijs van der Heijden for technical advice. This work is part of the research programme on Computational Sciences for Energy Research (14CSER005), which is financed by the Netherlands Organization for Scientific Research (NWO) and Royal Dutch Shell, and of the research programme on Marginal Soft Matter (FOM12CSM01), which is also financed by NWO.

\section{APPENDIX: AFFINE DEFORMATION WITH SSS}

In this appendix we demonstrate that, with our choice of spring constants $k_{i}=\frac{Y_{i}}{\ell_{\text {ca, }},}$, and in free-standing frames, the effect of engaging the state of self-stress on the total energy of an affine deformation is independent of strain, that is,

$$
\mathcal{E}(\Pi)-\mathcal{E}(0)=\mathcal{C}(\Pi) .
$$

The proof amounts to showing that both terms in Eq. (21) that depend on the displacements of the nodes are proportional to the overall stress in the frame, which is zero in free-standing frames since there are no external forces acting on them. In general, the Cauchy stress tensor for these frames is

$$
\sigma_{\alpha \beta}=\frac{\Pi}{V} \sum_{k=1}^{N_{b}} \ell_{0, k} t_{\mathrm{ss}, k} \hat{n}_{k, \beta} \hat{n}_{k, \alpha},
$$

with $V$ the system volume. By definition, it is zero for all values of $\Pi$, which means the sum itself must equal zero. Note that nothing below assumes that the displacements or the strains are small, so the result will be valid for nonlinear deformations.

The change in the length of any bond, $\delta l=\ell-\ell_{0}$, can be written as the displacement of the nodes that it joins. $\vec{u}_{i j}$ is the displacement vector, and $\vec{r}_{i}, \vec{r}_{j}$ are the position vectors to the $i$ th and $j$ th node such that $\ell_{0} \hat{n}=\vec{r}_{j}-\vec{r}_{i}$,

$$
\vec{u}_{i j}=\Lambda \cdot\left(\vec{r}_{j}-\vec{r}_{i}\right)-\mathbb{1}_{2}\left(\vec{r}_{j}-\vec{r}_{i}\right)=\left(\Lambda-\mathbb{1}_{2}\right) \cdot\left(\vec{r}_{j}-\vec{r}_{i}\right),
$$

where $\Lambda$ is the displacement gradient tensor. The fact that we are considering affine deformations corresponds to the application of this global tensor at the level of individual bonds. Using Einstein notation,

$$
\begin{aligned}
u_{\|} & =\hat{n} \cdot \vec{u}_{i j} \\
& =\hat{n}_{\alpha} \cdot\left(\vec{u}_{i j}\right)_{\beta}=\ell_{0}\left(\Lambda-\mathbb{1}_{2}\right)_{\alpha \beta} \hat{n}_{\beta} \hat{n}_{\alpha} .
\end{aligned}
$$

Thus, we can write the linear term (in $u_{\|}$) in Eq. (21) as

$$
\begin{aligned}
\sum_{k=1}^{N_{b}} t_{\mathrm{ss}, k} u_{k, \|} & =\left(\Lambda-\mathbb{1}_{2}\right)_{\alpha \beta} \sum_{k=1}^{N_{b}} \ell_{0, k} t_{\mathrm{ss}, k} \hat{n}_{k, \beta} \hat{n}_{k, \alpha} \\
& \sim\left(\Lambda-\mathbb{1}_{2}\right)_{\alpha \beta} \sigma_{\alpha \beta},
\end{aligned}
$$

which is zero in free-standing frames.

Secondly, consider the identity

$u_{\|}^{2}+u_{\perp}^{2}=\vec{u}_{i j} \cdot \vec{u}_{i j}=\ell_{0}\left(\Lambda-\mathbb{1}_{2}\right)_{\mu \alpha} \hat{n}_{k, \alpha} \ell_{0}\left(\Lambda-\mathbb{1}_{2}\right)_{\mu \beta} \hat{n}_{k, \beta}$.

Using this identity, the quadratic term in Eq. (21) can be written as

$$
\begin{aligned}
& \sum_{k=1}^{N_{b}}\left(\frac{t_{\mathrm{ss}, k}}{\ell_{0, k}}\right)\left(u_{k, \|}^{2}+u_{k, \perp}^{2}\right) \\
& =\sum_{k=1}^{N_{b}}\left(\frac{t_{\mathrm{ss}, k}}{\ell_{0, k}}\right) \ell_{0}\left(\Lambda-\mathbb{1}_{2}\right)_{\mu \alpha} \hat{n}_{k, \alpha} \ell_{0}\left(\Lambda-\mathbb{1}_{2}\right)_{\mu \beta} \hat{n}_{k, \beta} \\
& =\left(\Lambda-\mathbb{1}_{2}\right)_{\mu \alpha}\left(\Lambda-\mathbb{1}_{2}\right)_{\mu \beta} \sum_{k=1}^{N_{b}} \ell_{0, k} t_{\mathrm{ss}, k} \hat{n}_{k, \alpha} \hat{n}_{k, \beta} \\
& \quad \sim\left(\Lambda-\mathbb{1}_{2}\right)_{\mu \alpha}\left(\Lambda-\mathbb{1}_{2}\right)_{\mu \beta} \sigma_{\alpha \beta} .
\end{aligned}
$$

Again, this term is zero for free-standing frames, completing the proof.
[1] K. Bertoldi, V. Vitelli, J. Christensen, and M. van Hecke, Nat. Rev. Mater. 2, 17066 (2017).

[2] A. A. Zadpoor, Mater. Horizons 3, 371 (2016).

[3] P. M. Reis, H. M. Jaeger, and M. Van Hecke, Extreme Mech. Lett. 5, 25 (2015).

[4] K. E. Evans and A. Alderson, Adv. Mater. 12, 617 (2000).
[5] Y. Prawoto, Comput. Mater. Sci. 58, 140 (2012).

[6] D. R. Reid, N. Pashine, J. M. Wozniak, H. M. Jaeger, A. J. Liu, S. R. Nagel, and J. J. de Pablo, Proc. Natl. Acad. Sci. (USA) E1384 (2018).

[7] J. W. Rocks, N. Pashine, I. Bischofberger, C. P. Goodrich, A. J. Liu, and S. R. Nagel, Proc. Natl. Acad. Sci. (USA) 114, 2520 (2017). 
[8] S. Babaee, J. Shim, J. C. Weaver, E. R. Chen, N. Patel, and K. Bertoldi, Adv. Mater. 25, 5044 (2013).

[9] T. Frenzel, M. Kadic, and M. Wegener, Science 358, 1072 (2017).

[10] H.-H. Huang, B.-L. Wong, and Y.-C. Chou, Phys. Status Solidi B 253, 1557 (2016).

[11] M. Schenk and S. D. Guest, Proc. Natl. Acad. Sci. (USA) 110, 3276 (2013).

[12] J. T. Overvelde, T. A. De Jong, Y. Shevchenko, S. A. Becerra, G. M. Whitesides, J. C. Weaver, C. Hoberman, and K. Bertoldi, Nat. Commun. 7, 10929 (2016).

[13] C. Lv, D. Krishnaraju, G. Konjevod, H. Yu, and H. Jiang, Sci. Rep. 4, 5979 (2014).

[14] J. L. Silverberg, A. A. Evans, L. McLeod, R. C. Hayward, T. Hull, C. D. Santangelo, and I. Cohen, Science 345, 647 (2014).

[15] C. Coulais, A. Sabbadini, F. Vink, and M. van Hecke, Nature (London) 561, 512 (2018).

[16] C. Coulais, E. Teomy, K. de Reus, Y. Shokef, and M. van Hecke, Nature (London) 535, 529 (2016).

[17] X. Zheng, H. Lee, T. H. Weisgraber, M. Shusteff, J. DeOtte, E. B. Duoss, J. D. Kuntz, M. M. Biener, Q. Ge, J. A. Jackson et al., Science 344, 1373 (2014).

[18] T. A. Schaedler, A. J. Jacobsen, A. Torrents, A. E. Sorensen, J. Lian, J. R. Greer, L. Valdevit, and W. B. Carter, Science 334, 962 (2011).

[19] J. Gong, Y. Katsuyama, T. Kurokawa, and Y. Osada, Adv. Mater. 15, 1155 (2003).

[20] J. P. Gong, Soft Matter 6, 2583 (2010).

[21] E. Ducrot, Y. Chen, M. Bulters, R. P. Sijbesma, and C. Creton, Science 344, 186 (2014).

[22] H. Tsukeshiba, M. Huang, Y. H. Na, T. Kurokawa, R. Kuwabara, Y. Tanaka, H. Furukawa, Y. Osada, and J. P. Gong, J. Phys. Chem. B 109, 16304 (2005).

[23] M. Huang, H. Furukawa, Y. Tanaka, T. Nakajima, Y. Osada, and J. P. Gong, Macromolecules 40, 6658 (2007).

[24] N. K. Singh and A. J. Lesser, Macromolecules 44, 1480 (2011).

[25] Z. H. Yang, A. T. Detwiler, and A. J. Lesser, J. Mater. Sci. 47, 4251 (2012).

[26] C. Calladine, Int. J. Solids Struct. 14, 161 (1978).

[27] J. Wilhelm and E. Frey, Phys. Rev. Lett. 91, 108103 (2003).

[28] E. M. Huisman and T. C. Lubensky, Phys. Rev. Lett. 106, 088301 (2011).

[29] T. C. Lubensky, C. L. Kane, X. Mao, A. Souslov, and K. Sun, Rep. Prog. Phys. 78, 073901 (2015).

[30] J. Paulose, B. G.-g. Chen, and V. Vitelli, Nat. Phys. 11, 153 (2015).
[31] M. F. J. Vermeulen, A. Bose, C. Storm, and W. G. Ellenbroek, Phys. Rev. E 96, 053003 (2017).

[32] D. M. Sussman, C. P. Goodrich, and A. J. Liu, Soft Matter 12, 3982 (2016).

[33] R. Connelly and W. Whiteley, SIAM J. Discrete Math. 9, 453 (1996).

[34] S. Guest, Int. J. Solids Struct. 43, 842 (2006).

[35] S. D. Guest, IMA J. Appl. Math. 76, 57 (2011).

[36] M. Schenk, S. Guest, and J. Herder, Int. J. Solids Struct. 44, 6569 (2007).

[37] M. Wyart, Ann. Phys. Fr. 30, 1 (2005).

[38] D. J. Jacobs and M. F. Thorpe, Phys. Rev. Lett. 75, 4051 (1995).

[39] G. Düring, E. Lerner, and M. Wyart, Phys. Rev. E 89, 022305 (2014).

[40] J. Maxwell, Philos. Mag. 27, 294 (1864).

[41] K. D. Snelson, Continuous Tension, Discontinuous Compression Structures, US Patent 3169611 (1965).

[42] L. Henneberg, in Die Graphische Statik der Starren Systeme, B.G. Teubners Sammlung von Lehrbüchern auf dem Gebiete der Mathematischen Wissenschaften (Teubner, Leipzig, Berlin, 1911).

[43] W. G. Ellenbroek, M. van Hecke, and W. van Saarloos, Phys. Rev. E 80, 061307 (2009).

[44] C. P. Broedersz, X. Mao, T. C. Lubensky, and F. C. MacKintosh, Nat. Phys. 7, 983 (2011)

[45] S. Alexander, Phys. Rep. 296, 65 (1998).

[46] M. Wyart, L. E. Silbert, S. R. Nagel, and T. A. Witten, Phys. Rev. E 72, 051306 (2005).

[47] M. van Hecke, J. Phys.: Condens. Matter 22, 033101 (2010).

[48] A. J. Liu and S. R. Nagel, Nature (London) 396, 21 (1998).

[49] A. R. Cioroianu, E. M. Spiesz, and C. Storm, J. Mech. Phys. Solids 89, 110 (2016).

[50] Q. Wen, A. Basu, P. A. Janmey, and A. G. Yodh, Soft Matter 8, 8039 (2012).

[51] H. M. James, J. Chem. Phys. 15, 651 (1947).

[52] M. Wyart, H. Liang, A. Kabla, and L. Mahadevan, Phys. Rev. Lett. 101, 215501 (2008).

[53] P. R. Onck, T. Koeman, T. van Dillen, and E. van der Giessen, Phys. Rev. Lett. 95, 178102 (2005).

[54] B. Florijn, C. Coulais, and M. van Hecke, Phys. Rev. Lett. 113, 175503 (2014).

[55] A. Sydney Gladman, E. A. Matsumoto, R. G. Nuzzo, L. Mahadevan, and J. A. Lewis, Nat. Mater. 15, 413 (2016).

[56] M. Bai, A. R. Missel, W. S. Klug, and A. J. Levine, Soft Matter 7, 907 (2011).

[57] J. Fern and R. Schulman, Nat. Commun. 9, 3766 (2018). 